\section{Ausbildungsabbrüche aus gesundheitlichen Gründen seit 1985}

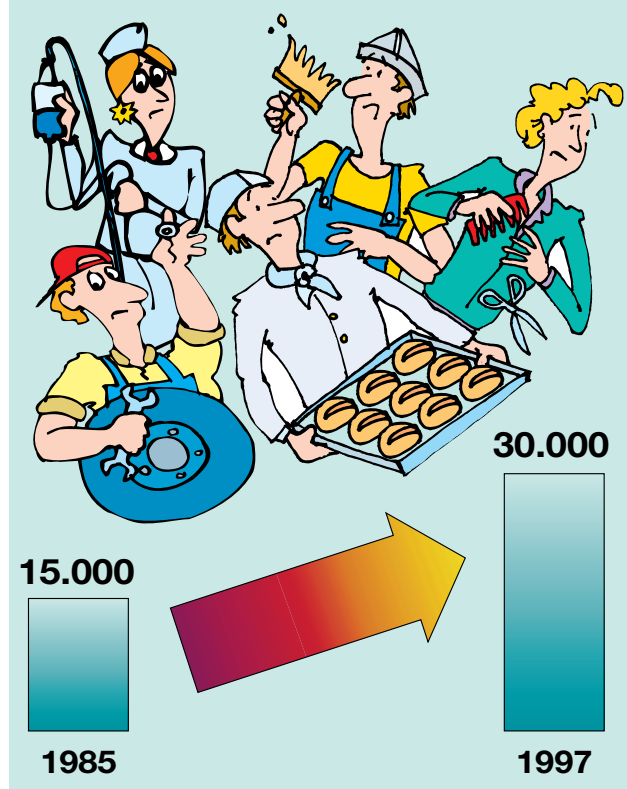

Die Zahl der Ausbildungsabbrüche hat sich seit 1985 verdoppelt. Als Hauptursache gilt die Zunahme von Hautkrankheiten durch unsachgemäßen Umgang mit Gefahrstoffen.

Die Prävention von Hauterkrankungen im Friseurhandwerk ist seit Jahren ein Schwerpunkt bei der BGW. Und das mit Erfolg. Die Zahl der bei der BGW gemeldeten Hauterkrankungen reduzierte sich von 4419 im Jahre 1991 auf 1824 in 1997. Auch bei den Auszubildenden ist der Trend positiv. Heute leiden $13 \%$ weniger Friseurlehrlinge an einer leichten bis schweren Hauterkrankung als vor zehn Jahren. Wollte deshalb 1987 noch jeder fünfte seine Ausbildung abbrechen oder nach der Lehre nicht in seinem Beruf weiterarbeiten, beabsichtigen heute nur noch 16\% die Aufgabe ihrer Ausbildung.

„Unsere Studie hat gezeigt, daß bei einer konsequenten Anwendung von Schutzmaßnahmen jeder das Gesundheitsrisiko im Friseurberuf minimieren kann, egal welcher Hauttyp vorliegt.“, erläutert Albrecht Liese, Leiter der Abteilung Prävention bei der BGW. Jugendlichen empfiehlt die BGW dennoch bereits vor der Wahl ihres Wunschberufes sich von einem Hautarzt beraten zu lassen. Hinweise auf eine eventuelle Veranlagung können auch Allergien in der Familie sein. „Wer sich so auf den Berufsstart vorbereitet, hat sein Erkrankungsrisiko bereits deutlich verringert", erklärt Liese weiter.

Quelle: Berufsgenossenschaft für Gesundheitsdienst und Wohlfahrtspflege (BGW).

\title{
Arbeitsunfälle und Berufskrankheiten bei der BGW stark gesunken
}

\section{Die Zahl der Arbeitsunfälle und Berufskrankheiten bei den fünf Mil- lionen Versicherten der Berufsgenossenschaft für Gesundheitsdienst und Wohlfahrtspflege (BGW) ist 1997 deutlich gesunken. Mit rund 86000 gemeldeten Fällen sank die Zahl ge- genüber dem Vorjahr um 7,8\% bei einem gleichzeitigen Zuwachs der Mitglieder um 20000 Unternehmen auf über 440000.}

$\mathrm{D}$ as ist ein deutlicher Trend, der zeigt, daß unsere Präventionsmaßnahmen greifen", kommentiert BGW-Hauptgeschäftsführer G. Mehrtens das positive Ergebnis. So gab es bei den meldepflichtigen Arbeitsunfällen einen Rückgang von $6,8 \%$ auf rund 51600 Fälle. Bei den Berufskrankheiten gingen die Verdachtsfälle um rund $3 \%$ auf rund 11250 zurück, und bei den Wegeunfällen wurde gar ein Rückgang um $12 \%$ auf rund 23000 Fälle registriert.

Stolpern, Stürzen und Ausrutschen sind mit 37\% die Hauptursachen für Arbeitsunfälle. In 34\% der gemeldeten Fälle haben sich die Beschäftigten gestoßen, und bei jedem fünften Unfall wurden Arbeitnehmer von Gegenständen getroffen. $43 \%$ aller Wegeunfälle Unfälle zwischen Arbeitsplatz und Wohnung - ereigneten sich erwartungsgemäß vor allem in den Wintermonaten von November bis Februar.

Beim Verdacht auf eine Berufskrankheit stehen Hauterkrankungen mit 53\% weiterhin an erster Stelle, gefolgt von Wirbelsäulenerkrankungen mit 18,9\% und Atemwegserkrankungen mit $10,7 \%$.

Besonders erfreulich ist der anhaltend positive Trend bei Hauterkrankungen im Friseurhandwerk. Hier wurden gegenüber dem Vorjahr 204 Fälle weniger registriert, ein Rückgang von gut $10 \%$. Die vierte große Gruppe der Berufskrankheiten bilden die Infektionskrankheiten mit 8,6\%. Hier sind wie auch schon im Vorjahr Hepatitis B und Hepatitis $\mathrm{C}$ die am häufigsten auftretenden Erkrankungen.

Ein Problem sind Haut- und Atemwegserkrankungen durch Naturlatexallergien, die von 944 Fällen im Jahre 1996 auf 1211 in 1997, d.h. um rund $28 \%$ zunahmen. Betroffen sind dabei vor allem Arzthelferinnen und Krankenschwestern. Die BGW startete zu diesem Thema im letzten Jahr eine Schwerpunkt-Kampagne, mit dem Ziel, auf Latex als Gefahrstoff aufmerksam zu machen. „Unsere laufende Kampagne gegen Latexallergie hat die Versicherten für dieses Thema sensibilisiert", erklärt Gerhard Mehrtens. „Daher hat es hier zunächst einen Anstieg der Meldungen gegeben“. Ein Phänomen, das häufig nach Aufklärungskampagnen im Präventionsbereich auftritt. „Für dieses Jahr erwarten wir allerdings einen Rückgang, da sich die empfohlenen Schutzmaßnahmen in der Praxis immer mehr durchsetzen“, so Mehrtens weiter.

Quelle: Berufsgenossenschaft für Gesundheitsdienst und Wohlfahrtspflege (BGW). 